\title{
Philosophiques
}

\section{Signification et états mentaux : à propos de l'" antireprésentationnalisme » de Wittgenstein}

\section{Denis Sauvé}

Volume 25, numéro 1, printemps 1998

URI : https://id.erudit.org/iderudit/027470ar

DOI : https://doi.org/10.7202/027470ar

Aller au sommaire du numéro

Éditeur(s)

Société de philosophie du Québec

ISSN

0316-2923 (imprimé)

1492-1391 (numérique)

Découvrir la revue

Citer cet article

Sauvé, D. (1998). Signification et états mentaux : à propos de l'« antireprésentationnalisme » de Wittgenstein. Philosophiques, 25(1), 29-48. https://doi.org/10.7202/027470ar
Résumé de l'article

Wittgenstein, selon R. Rorty, accepte dans ses Recherches philosophiques une variété d' « antireprésentationnalisme » en ce sens qu 'il refuse la distinction entre certaines représentations envers lesquelles on devrait adopter une attitude réaliste et d'autres envers lesquelles il faudrait adopter une attitude non réaliste (ou antiréaliste). Je soutiens dans cet article que le contraire est vrai. Wittgenstein adhère en particulier à une forme de non-réalisme quant au concept de signification et certains concepts d'états et de processus mentaux. L'expression " la signification de $\mathrm{M}$ » $\mathrm{n}$ 'a pas selon lui une fonction reférentielle (il n 'existe pas une " chose " appelée « la signification de M » en vertu de laquelle « $M$ » serait douée de signification). Une expression du vocabulaire mental telle que " savoir réciter l'alphabet» ne désigne pas un état interne (au sens de la psychologie ou de la physiologie) de l'individu sachant réciter l'alphabet. «Savoir réciter l'alphabet » et « la signification de $M$ » ne sont pas des expressions employées pour " parler de » quelque chose ou s'y référer. 


\title{
SIGNIFICATION E ÉTAIS MENTAUX : À PROPOS DE L'“ ANIREPRÉSENIATIONAALISME » DE WIIIGENSIEIN
}

PAR

DenIS Sauvé

\begin{abstract}
Si jünais à dire quelle est lerreur principale commise par les philosophes de la présente génération (.../,je dirais qu elle réside en ceci que, lorsque l'on regnrde le langage, ce sont les formes d'expression que l'on regarde et non l'usage que lon fait de ces formes d'expression.
\end{abstract}

Wittgenstein

RÉSUMÉ : Wittgenstein, selon R. Rorty, accepte dans ses Recherches philosophiques une variété d' antireprésentation. nalisme " en ce sens qu'il refuse la distinction entre certaines représentations envers lesquelles on devrait adopter une attitude réaliste et d'autres envers lesquelles il faudrait adopter une attitude non réaliste (ou antiréaliste). Je soutiens dans cet article que le contraire est vrai. Wittgenstein adhère en particulier à une forme de non-réalisme quant au concept de signification et certains concepts d'états et de processus mentaux. L'expression "la signification de $M$ " n'a pas selon lui une fonction référentielle (il n'existe pas une "chose "appelée "la signification de $M$ " en vertu de laquelle " $M$ " serait douée de signification). Une expression du vocabulaire mental telle que "savoir réciter l'alphabet "ne désigne pas un état interne. (au sens de la psychologie ou de la physiologie) de l'individu sachant réciter l'alphabet. "Savoir réciter l'alphabet " et " la signification de $M$ " ne sont pas des expressions employées pour "parler de "quelque chose ou s'y référer.

ABSTRACT: R. Rorty claims that Wittgenstein agrees in the Philosophical Investigations with "antirepresentationalism" in that he rejects the distinction between representations toward which we should have a realist attitude and those toward which we should have the opposite, nonrealist, attitude. The aim of this paper is to show that this reading is incorrect. Wittgenstein accepts antirealist views in particular with respect to meaning and (at least) some mental 
concepts. The expression "the meaning of $W$ ", he holds, is not a referential expression (there is nothing which we may call "the meaning of $W$ " in virtue of which " $W$ " has that meaning). Expressions from our mental wocabulary such as "knowing the $A B C$ " do not refer to internal states (like those studied by psychology. or neurophysiology). "The meaning of $W$ " and "knowing the $A B C$ " are not expressions used to refer to or "talk about" anything.

Richard Rorty range le second Wittgenstein, l'auteur des Recherches philosophiques, parmi ces philosophes qui, dans les diseussions sur la question du réalisme, défendent ce qu'il appelle l'c antireprésentationnalisme ». Un problème qui est soulevé dans ces (liscussions est, dit Rorty, celui de savoir " quelles sortes d'énoncés vrais, s'il y en a, entretiennent des relations de représentation [ou de correspondance] à des items non linguistiques" ${ }^{1}$. Les "représentationnalistes " pensent que de tels énoncés existent ; ils font cn conséquence une distinction entre les énoncés vrais qui correspondent à (ou sont "rendus vrais "par) une réalité indépendante el vis-à-vis desquels ils adoptent une attitude "réaliste ", et ceux qu'on pourrait qualifier de « vrais » mais qui n'entretiennent pas une telle relation à des états de choses indépendants, des énoncés vis-à-vis desquels ils recommandent une attitude " non réaliste " ou "antiréaliste ". Les "antireprésentationnalistes " sont ceux qui mettent en doute la pertinence de la distinction. Les notions de correspondance et de représentation sont selon eux des notions dont on doit se passer " non seulement [comme pour les représentationnalistes] en regard d'une certaine classe [...] d'énoncés, mais en regard de tous les énoncés ${ }^{2}$. Pour Rorty, le Wittgenstein de la première période, l'auteur du Tractatus, faisait partie du groupe des "représentationnalistes " alors qu'il est devenu durant la seconde période de sa vie philosophique un « antireprésentationnaliste ». Je pense quion peut hui donner raison sur le premier point mais non sur le second.

Jc défendrai dans ce qui suit l’interprétation suivante: Wiltgenstein préconise dans les Recherches une attitude "non réaliste" vis-à-vis deux types de concepts qui occupent une place centrale dans sa seconde philosophie, soit le concept de signification et le concept d'état mental (ou différents concepts d'états ou de processus mentaux). On peut dire en ce sens qu'il adopte un point de vue "réaliste n à l'égard (disons) des concepts de chaise ou d'animal. mais une forme de "non-réalisme "à l'égard des concepts de signification et de certains concepts mentaux. Si c'est le cas, il semble

1. Richard Rorty, Objectivity, Relativism and Truth, Philosophical Papers, Vol. 1, Cambridge, Cambridge University Press, 1991, p. 2.

2. Ibid., p. 4 . 
que, comme le Wittgenstein du Tractatus, il s'intéresse au problème des engagements ontologiques du langage, c'est-à-dire au type de questions dont s'occupent les représentationnalistes, en dépit des différences - dont il ne sera pas question ici - qui le séparent du Wittgenstein de la première période.

Pour défendre cette interprétation, j’esquisserai dans la premièro section larrière-plan de ses remarques sur les concepts de signification et d'état mental. La seconde portera sur le concept de signification et la troisième sur quelques concepts mentaux traités dans les Recherches ${ }^{3}$.

\section{Les nombres, le temps, l'esprit, lieu}

On comprend l'importance du phénomène du langage et de la notion de signification dans sa seconde philosophie si on se rappelle la façon dont Wittgenstein définit son projet philosophique: la tâchc qu il assigne à la philosophie est d'éliminer les "méprises " el les " confusions" auxquelles nous sommes sujets ( nous" cest-à-dire en particulier les philosophes ou, peut-être, les "métaphysiciens "), des méprises dont la source est le langage. On lit par exemple dans le Cahier bleu : La philosophie, au sens où nous employons le mol. est un combat contre la fascination qu'exercent sur nous [nos] formes d'expression " $(B B$, p. 27). Dans les Recherches, on lit : la philosophic est « un combat contre l'ensorcellement de notre intelligence au moyen du langage " $(P U, \S 109)^{4}$.

Quelles sont ces méprises? Les méprises se produisent quand on cente de répondre à des questions telles que: "Qu'est-ce qu'un nombre?" ", Qu’est-ce que le temps?" " "Qu'est-ce que la pensée?" ". "Qu'est-ce que l'esprit? "ou "Quel genre d'être est Dien ? ” .- des questions qui ont l'air parfaitement sensées (si on peut demander "Qu'est-ce quun arbre?", pourquoi pas également "De quelle nature sont les nombres?") mais des questions dans lesquelles les formes d'expression elles-mêmes, les mots " pensée ", "temps " ou " nombre ", se révèlent sources de confusion : "lorscfue nous nous

3. Les abréviations pour les références aux nuvres de Wittgenstein seront les suivantes : $T$ pour Tractatus Logico-Philosophicus (Londres, Routledge and Kegan Paul, 1961) ; $P U$ pour Philosophische Untersuchungen, éd. par G. E. M. Anscombe et R. Rhees (Francfort, Suhrkamp, 1969); $B B$ pour The Blue and the Brown Books (Oxford, Blackwell, $2^{e}$ éd., 1969) ; WLC pour Wittgenstein's Lectures, Cambridge 1932-35, éd. par A. Ambrose (Chicago, University of Chicago, 1982); $P G$ pour Philosophische Grammatik, édl. par R. Rhees (Francfort, Suhrkamp, 1969); $Z$ pour Zettel, éd. par G. E. M. Anscombe et G. H. von Wright (Berkeley et Los Angeles, University of California Press, 1967); BPPI pour Bemerkungen über die Philosophie der Psychologie, vol. 1, éd. par G. E. M. Anscombe et G. H. von Wright (Oxford, Blackwell, 1980); BPPII pour Bemerkungen über die Philosophie der Psychologie, vol. 2, éd. par G. H. von Wright et H. Nyman (Oxford, Blackwell, 1980) ; LC pour Lectures and Conversations on Aesthetics, Psychology and Religious Belief, éd. par C. Barrett (Berkeley et Los Angeles, University of California Press, 1972).

4. Il dit également dans l'un de ses cours : " nos investigations portent sur le langage et sur les énigmes (puzzles) qui surgissent de l'usage du langage "(WLC, p. 31). 
préoccupons au sujet de la nature de la pensée, la perplexité que nous interprétons incorrectement comme portant sur la nature d'un médium [l'esprit considéré comme médium de la pensée] est une perplexité causée par l'usage mystifiant du langage " $(B B, p, 6)$. Ce genre d'erreur « réapparaît continuellement en philosophie, lorsque par exemple nous sommes dans l'embarras à propos de la nature du temps, lorsque le temps nous semble une chose bizarre "(Ibid.). C'est alors "l'usage du substantif " temps" qui nous mystifie "(Ibid.). Et "[c]e c|ui se produit avec les mots "Dieu" et "âme" [ou " temps", "pensée " et "esprit"] est ce qui se produit avec le mot " nombre ". Même si nous renonçons à expliquer ces mots par ostension [par le recours à une définition "ostensive"), en pointant [vers quelque chose |, nous ne renonçons pas à les expliquer en termes substantifs » (WLC, p. 32). Comme “ il n'y a pas dobjet correspondant [au substantif" "le nombre un "| au sens où il y a un objet correspondant [au nom] "Smith", nous nous mettons en quête d'un objet en un autre sens " (WLC, p. 14).

On lit dans les Recherches: "Nos considérations éclairent |les problèmes philosophiques] en écartant les méprises [...] concernant lusage de mots causées entre autres par certaines analogies entre les formes d'expression dans différentes régions du langage " $(P U, \S 90)$. Les méprises ont leur source dans l'analogic entre les expressions "lcmps", " esprit», " nombre un , etc. et les expressions qui lénotent des objets concrets : "Un substantif dans la langue est d'abord [primarily] employé pour désigner un objet physique et un verbe pour désigner un mouvement d'un tel objet "(WLC, p. 119). On tend à penser que le temps (les esprits, le nombre un) existe dans la mesure où il y a des tables (des animaux, etc.) : si le mot "table " est employé pour parler du type d'objet appelé “ table "en français (pour s'y référer), il semble que ce soit vrai également du mot "lemps" par conséquent. le temps existe et il est légitime de soulever la question de sa nature. On peut en dire autant du verbe "penser" : "l'existence [du verbe] " penser" [...] à côté des mots dénotant des activités corporelles telles qu'écrire, parler, etc. nous incite à chercher une activité, différente de ces activités mais analogue à celles-ci, correspondant au mot " penser" " $(B B, p .7)$. On no lient pas compte de: la différence entre l"usage (ou la grammaire) des mots "temps". "pensée " ou " nombre ", d'un côté et, de lautre. lusage des mots référant à des objets concrets ou à leurs propriétés. La tâche du philosophe est de mettre justement en lumière ces différences dans le but d'éliminer les problèmes philosophiques : “ Nous nous débarrassons de la perplexité qui s'attache à fla question "Qu'est-ce qu'un nombre?" en clarifiant nos idées au sujet de la grammaire du mot " nombre " [...]. En mettant au clair nos idées à propos de l'usage du mot " nombre ", nous cessons de poser la question "Qu'est-ce qu'un nombre?" "(WLC, p. 164). On trouve dans les Recherches une remarque similaire au sujet du concept de compréhension (à propos de verbes psychologiques comme 
" comprendre un mot" ou "comprendre comment suivre une règle s): quand on se méprend sur "l'usage d'un mot [par exemple du verbe "comprendre "|, on l"interprète comme l'expression d"un processus étrange (Comme on s'imagine le temps comme un médium étrange ou l'esprit comme une chose étrange) 》 $(P U, \$ 196)$.

Wittgenstein ne nous dit pas ce quil faut entendre ici plus exactement par l" "usage " ou la " grammaire "d'un mot, mais il semble qu il veuille dire au moins ceci : si on comprend la grammaire de lexpression "le nombre un ", on cesse de poser la question "Qu'est-ce que le nombre un?" dans la mesure où celle-ci montro qu'il n'y a pas une sorte d' objet éthéré " (WLC, p. 44) - le nombre un - auquel l'expression aurait pour rôle de référer. Le fait qu’on me puisse définir l"expression " par ostension ", au sens où il peut y avoir une "définition ostensive " des mots " table " ou " rouge ", est un trait de sa " grammaire ». On peut expliquer à quelqu un ce que " trois" signifie en lui montrant des groupes d'objets au nombre de trois (voir IWLC, p. 45), mais on ne définit pas " trois" en montrant le nombre trois: "Il ny a pas davantage de processus de monstration lié à lexplication du mot "nombre", qüil y en a à l'explication de l'expression " la permission de s'asseoir sur" un siège au cinéma" " (WLC, p. 32). C"est vrai également de certains verlues désignant. suivant la grammaire courante, des "ćtats "ou des "processus" psychologiques: si on a saisi correctement " "usage "ou la "grammaire" - au sens de la "grammaire philosophique "- du verbe "comprendre" (entre autres expressions du vocabulaire mental), on peut voir que "comprendre " n'esl pas employé pour rlésigner quoi que ce soit, ni un état, ni une activité ou un processus psychologique (je reviens plus loin sur ce point).

Il existe une autre source de confusions au sujet des mols " nombre ", "temps" ou "pensée " lorsque les philosophes posent des questions telles que "Qu'est-ce qu'un nombre?" ou “Qu'est-ce que le temps? m. Elle réside dans une conception erronéc de la signification que les questions présupposent : "lorsqu'ils entendent. le substantif "le nombre un ", les gens ont tendance à concevoir sa signification comme se trouvant au-delà du signe et comme lui correspondant, de la façon dont Smith correspond au nom "Smith " (WLC, p. 44). On suppose que, pour qu'un mot " possède" une signification, il doit exister quelque chose en quoi consiste cette signification, par exemple le temps, cette réalité à laquelle on réfère quand on emploie le mot "temps". Lorsque nous recherchons "la signification d'un mot ", " nous la recherchons comme s'il s'agissait d'un objet coxistant avec le signe" et une raison pour laquelle " nous commettons cette erreur est [...] que nous nous mettons à la recherche d'une " chose correspondant à un substantif" " $(B B, \mathrm{p} .5)$. Nous considérons les mots " comme s’ils étaient des noms propres, et nous confondons alors le porteur du nom avec la signification du 
nom $\gg(B B, \text { p. } 18)^{5}$. Parfois, on pense que la chose qui « coexiste avec le signe „ est non pas un objet (le porteur du nom s'il en a un) mais une image mentale (" une image ou une chose qui est mise en corrélation avec le mot " (BB, p. 181) ou bien un " sens ", une pensée ou un concept. On voit dans l'expression " la signification de " $M$ " "une expression référentielle, laquelle doit par conséquent désigner "quelque chose ", une chose que l'on assimile ensuite à un objet (le porteur (le " $\mathrm{M} »)$, à une entité psychique (une image, une sensation ou une émotion) ou bien à un objet abstrait (un sens frégéen ou un concept).

Wittgenstein fait ici deux suggestions : 1) au lieu de s'intéresser à "la signification " l'un mol, on devrait s'intéresser à « l'explication de la signification d'un mot", et 2) plutôt que de parler de la signification d'un mot, on revrait parler de son "usage ".

On lit dans le Cahier bleu: "Dit sommairement," demandonsnous ce qu'est l'explication de la signification, car peu importe ce que cela cxplique, ce sera la signification" ensuite: "ELudier la grammaire de l'expression "explication de la signification" va vous enseigner quelque chose à propos de la grammaire du mot "signification " et vous guérir de la tentation de chercher un objet que vous pourriez appeler "la signification" " (Ibid.). Étudier la grammaire de l'expression " la signification d'un mol" (sa " grammaire philosophique »), c'est-à-dire son « usage ». montre qu'il n'y a pas " quelque chose " qu'on pourrait appeler "la signification d'un mot ". Si on examine le genre de contexte dans lequel les gens parlent de la signification d'un mot (ou de la signification d'une expression ou d'une phrase). on voit que " signification " s'emploie pour dire des choses telles que " $\mathrm{Ce}$ mot a la même signification que celui-là "ou "Ce mot est dénué de signification ". mais ces façons de parler ne nous engagent pas vis-à-vis l'existence d'entités particulières appelées des a significations ". Wittgenstein dit dans un cours : "Les idées de signification et de sens sont périmées |obsolete]. Sauf si " sens " est utilisé dans des phrases comme " Cela n’a pas de sens "ou "Ceci a le même sens que cela ". nous ne nous intéressons pas à [la notion] de sens » (WLC, p. 30). Mais. d'autre part, il soutient également que les explications de la signification ne sont en fait rien d'autre que des explications de l"usage: "S"il peut être difficile d'expliquer " Iongueur" mais non pas d'expliquer" mesure d'une longueur". de façon analogue il est moins difficile de décrire co quion appelle " explication de la signification "que d'expliquer" " signification" " (WLC, p. 48). Et il ajoute immédiatement après: "On explique la signification d'un mot en décrivant son usage " (Ibid., ; voir aussi $P G$, p. 59). Ainsi. il semble que la suggestion 1) se ramène à la suggestion 2) : si on examine la manière dont on s'y

5. L'erreur était celle de Wittgenstein lui-même dans le Tractatus, là où il écrivait : “ [l]e nom signifie l'objet. L'objet est sa signification " $(T, 3.203)$. 
prend pour expliquer la signification des mots - si, autrement dit, on regarde la "grammaire" de l'expression "explication de la signification "- on remarque que ces explications se ramènent généralement à des descriptions de l'usage et, si on admet que la signification est l'usage, veut apparemment dire Wittgenstein, il n'es! plus nécessaire de postuler des "significations" en tant qu* "choses" correspondant à nos mots. Car "[u]tiliser l'expresssion " signification d"un mol " comme équivalente à "usage d"un mot "présente entre autres lavantage de nous montrer quelque: chose au sujet du cas philosophique bizarre oì nous parlons d"um objet [par exemple le nombre un] correspondant [à l'expression " le nombre un "]»(WLC, p. 44). Nous voyons qu’il n'est pas nécessairc de supposer que, pour qu'ils aient une signification, il doit correspondre aux mots " nombre ", "temps "ou "esprit "des choses telles que le temps, les nombres, etc. : il suffit qu'ils aient un usage. Il existe évidemment des mots, par exemple "Smith", auxquels "correspondent "des objets, à savoir leur porteur, mais si on est prêt à dire que la signification est l'usage, rela n'a pas à être vrai dans tous les cas: les expressions " temps ", " nombre ", "esprit "on " permission de s'asseoir sur un siège au cinéma" ont une signification. puisqu'elles ont un usage, mais il n'est pas plus indispensable pour qu’elles possèdent une signification qüil existe " quelque chose q qui leur correspond qu’il ne suit, de ce que les particules logiques " el ". "ne... pas " et " ou ont une signification, qu'il y a des choses lelles que la négation, la disjonction ou la conjonction - - des entités en vartu desquelles elles auraient cette signification. On lit dans le Cahier. bleu: il noest pas moins étonnant « que l’homme ait conçu une. divinité du temps qu’il ne l'aurait été [s'il avait conçu] une divinité de la négation ou de la disjonction » $(B B$, P. 6). Il aurait été étonnant, autrement dit, que nos ancêtres imaginent « une divinité de la négation ou de la disjonction ", croyant que " ne... pas " el " ou » jouent dans la langue le rôle de substantifs ; mais il n'est pas moins curicux que l'homme ait imaginé - puisqu'il a effectivement imaginé - une divinité du temps, pensant que a temps "joue dans la languc le rôle d'un substantif.

\section{La signification, l'usage ou le rôle des mots}

Deux questions en ce point se posent. La première a trait au statut du slogan " La signification est l'usage »: faut-il y voir une " analyse », une proposition de a révision "ou une analyse partielle du concept ordinaire de signification? Wittgenstein écrit : "Pour une large part des cas d'emploi du mot "signification" - encore que ce ne soit pas pour tous les cas - on peut l'expliquer ainsi : la signification d'un mot est son usage dans le langage " $(P U, \S 43$; voir $W L C$, p. 48). Il dit cependant peu de choses à ce sujet. Je supposerai, comme le suggère ce passage, qu'il propose une sorte d'analyse partielle du concept, peut-être aussi bien une " analyse " qu'une " révision " du concept. La seconde question - à laquelle je donnerai seulement une esquisse de. 
réponse - porte sur le contenu du slogan : que doit-on comprendre plus précisément par l'" usage "d'une expression " dans le langage "?

Les premiers paragraphes des Recherches traitent de ce que Wittgenstein appelle le " concept philosophique de la signification " $(P U, \S 2)$. Cette conception illustrée par la citation de Saint-Augustin revient à soutenir que " chacun des mots a une signification " et que cette signification est " coordonnée au mot, [étant] l'objet auquel le mot se rapporte " $(P U, \S 1)$. Comme dans le Tractatus, tous les mots, suivant la conception augustinienne du langage, " nomment des objets " (Ibid.) (des tables ou des chaises et peut-être des actions et des propriétés) et leurs significations sont les choses mêmes auxquelles réfèrent les mots. Wittgenstein décrit ensuite un jeu de langage (un emploi de signes et les activités concomitantes des locutcurs (voir $P U, \S 7$ et 321]) dans lequel un individu $\mathrm{A}$ demande à un autre $\mathrm{B}$ d'aller acheter des pommes : $A$ remet à $\mathrm{B}$ un bout de papier sur lequel sont écrits les mots "cinq pommes rouges"; B se rend à une épicerie et le montre à l'employé ; celui-ci se dirige ver's une armoire portant une étiquette sur laquelle on lit le mot " pomme "; il en retire, en comptant de un à cinq, cinq pommes de la même couleur que celle d'un échantillon de couleur rouge et il vient les remettre à $B$. L'exemple est apparemment destiné à montrer que tous les mots ne sont pas des noms (" cinq ", par exemple, ne joue pas dans le jeu le rôle d'un nom ou d'un substantif) et que ce que signifient les mots n'est pas "quelque chose" qui leur est " coordonné ", ni un objet dans le monde ni un objet " en un autre sens ", mais leur usage. Wittgenstein n'en dit cependant pas davantage ce qu'il faut comprendre par l' "usage "d'un mot. Plus loin, il compare les mots à des outils ou à des instruments; l' "usage " (la "fonction" ou la "grammaire") des noms de nombres est " totalement différent " de celui du mot « pomme „ $(P U, \S 10):$ « [l] es fonctions [les usages] des mots sont aussi diverses que les fonctions [d'outils comme une scie, un marteau ou un pot de colle]" $(P U$, $\$ 11$; voir $W L C$, p. 103$)^{6}$. Mais en quel sens?

On lit dans le Cahier bleu : on tend à oublier " que c'est seulement l'usage particulier d'un mot qui lui donne sa signification ". En effet, "|p]ensons à notre vieil exemple de l'usage de mots : quelqu'un est envoyé chez l'épicier avec un bout de papier sur lequel sont écrits les

6. On pourrait être tenté de dire que les "usages" ou "fonctions" des mots " pomme ", " couge " et " cinc " diffèrent au sens suivant: "pomme " a pour fonction de désigner une certaine catégorie d'objets (une sorte de fruit) alor's que "rouge " a pour fonction de désigner une certaine couleur et " cinq ", le cincpuième membre de la série des entiers naturels. Les mots "désignent " tous quelque chose et ne diffêrent que par le type de chose désignée (" chaise " et " temps " sont tous les deux des substantifs sauf qu'ils réfêrent à des choses d'espèces totalement différentes). Mais c'est précisément ce que nie Wittgenstein : " assimiler de cette façon [en disant qu'ils "désignent "tous quelque chose] les descriptions de l'usage des mots ne rend pas ces usages plus semblables les uns aux autres " $(P U$, $\$ 10)$. 
mots " cinq pommes" ". Ainsi, " [l]"usage d"un mot dans la pratique est sa signification " $(B B$, p. 69 ; voir $B B$, p. 16-17).

Supposons que nous modifions comme suit le jeu de langage du passage des Recherches (voir $B B$, p. 172-173) : au lieu d'un bout de papier" sur lequel est inscrite la phrase "cinq pommes rouges ». $A$ remet à $B$ un bout de papier sur lequel il a écrit : “ $\mathrm{X}$ - $\mathrm{N}$-cinq pommes rouges ». " $X$ " représente dans le jeu le nom d"une épicerie (on a enseigné à $B$ à s'y renclre quand il le lit). " $N$ » est lc nom d"un employé : lorsque $\mathrm{B}$ entre dans l'épicerie, il le prononce el $\mathrm{N}$ accourt (l’employé a été entraîné à agir ainsi toutes les fois qu'il contend prononcer son nom). B se rend donc à l'épicerie, il montre à $\mathrm{N}$ le. bout de papier et celui-ci va chercher cinc pommes rouges en agissant comme l'employé dans le jeu des Recherches. Wittgenstein pose maintenant la question : en quoi consiste la relation entre les noms et les choses dont ils sont les noms, par exemple entre " $X$ » el la maison? Une réponse serait que " la relation consiste en ce que certains traits ont été peints sur la porte de la maison » $(B B, p .172)$. Mais ce n'est qu'une partie de la réponse : " la relation |... est établic non pas seulement par le fait qu'on a peint ces traits sur la porte mais par le rôle particulier que [ceux-ci] jouent dans la pratique de notre langage tel que nous l'avons esquissé " (Ibid.). De façon similaire, on pourrait dire que a la relation du nom d'une personne à cette personne consiste en ce que celle-ci a été entraînée à accourir vers quelqu'un qui l’a appelée en prononçant son nom ; ou bien encore, nous pourrions dire qu'elle consiste en cela ainsi que dans la tolalité de l'usage du nom dans le jeu de langage » (Ibid. ; je souligne).

La question que Wittgenstein pose revient à demander : en quoi consiste la signification de " $\mathrm{X}$ " dans le jeu (la situation est beaucoup) plus complexe pour le cas d'une langue naturelle mais, semble dire Wittgenstein, elle n'est pas essentiellement différente.) ?' Autrement dit, en vertu de quoi "X " est-il précisément ce mot!" Ou encore : qu'est-ce qui est constitutif de son identité comme signe? La réponse qu"il suggère est que " $X$ " a cette signification (il est ce mol) en vertu de son usage, de la totalité de son usage dans le jeu, et par son "usage " (ou "usage dans la pratique ") on veut dire le lait que: $\Lambda$ ait inscrit " $X$ " sur un bout de papier, que $B$ lise le signe, que $B$ voic le signe peint sur la porte de l'épicerie (et le fait que quelqu'un l'ait peint auparavant sur la porte), que $B$ se dirige vers la maison sur la porte de laquelle il l'a vu, etc. On pourrait également demander : pourquoi le mot "rouge " est-il le nom de la couleur rouge? La réponse serait : "rouge " est le nom de cette couleur en vertu, entre autres, du fait que lorsque B montre à l'employé le bout de papier sur lequel sont écrits les mots a cinq pommes rouges ", il choisit des pommes précisément de cette couleur en utilisant le tableau sur lequel le mot est écrit face à l'échantillon. Comme on lit dans les Recherches, la relation entre le nom et l'objet "peut aussi consister, parmi beaucoup d'autres choses, dans le fait qu'entendre le 
nom invoque à notre esprit l'image de la chose qui le porte ; et cela consiste aussi, entre autres, en ce que le nom est inscrit sur la chose ou en ceci qu'il est prononcé lorsque la chose est indiquée du doigt » $(P U, \S 37$; je souligne). Des faits de ce genre sont constitutifs de la "relation" entre le nom et son porteur: le mot est le nom d'une chose (en général un mot a la signification qu'il possède) en vertu de faits relatifs aux activités des locuteurs quand ils "opèrent avec le mot " (voir $P U, \S 1$ ).

On trouve une idée semblable dans ce passage où il est question d'un " explorateur" qui entreprend de traduire une langue " qui [lui] esl complètement étrangère » $(P U, \S 206)$. La question qui se pose est rle savoir" " $\mathrm{d}]$ ans quelles circonstances [Umständen], [l'explorateur va pouvoir dire] que les gens qui [parlent la langue] donnent [par exemple] des ordres, les comprennent. refusent d'y obéir, etc.[...]" (Ibid.). La réponse est que a [la] façon d'agir commune des êtres humains est le système de référence au moyen duquel nous interprétons une langue étrangère "(Ibid.). L'explorateur (on dirait aujourd'hui l'" interprète radical ") doit d'abord comprendre le sens des activités rles locuteurs (en se référant à la "façon d'agir communc des êtres humains $n$ ), ce qui lui permettra ensuite d'interpréter leur langage. Il observe par exemple un " constructeur " $A$ et son aide $B$ en train d'ériger une maison (voir $P U, \S 2$ ). A émet parfois des sons auxquels $B$ réagit en effectuant certaines actions. L'explorateur constate entre autres " circonstances" que toutes les fois que $\mathrm{A}$ a besoin de briques, il émet un des sons ("Briques!») et que B agit en lui apportant une brique. Il en infère que l'énoncé revient à donner un ordre (voir $P U, \S 21$ ) et le traduit par la phrase "Apporte-moi des briques! ». Liénoncé du constructeur est un ordre étant donné les " circonstances" du jeu : il ne s'agirait pas d'un ordre si, entre autres circonstances, A n'avait pas besoin de briques, si, après l'avoir entendu, B n’allait pas la plupart du temps chercher des briques, si A n'était pas le patron et B son employé, etc. Sachant quelles sont les circonstances, le traducteur peut connaître l' "usage "(la "fonction " ou le "rôle ») de l'énoncé dans le jeu ( “ [C]e qui caractérise un ordre en tant que tel ou une description en tant que telle ou une question cu tant (que telle, etc. est [...] le rôle que [son] énonciation [...] joute (lans la pratique $|. .$.$| du langage. \triangleright \mid B B$, p. 102-103]). Les circonstances sont constitutives de la signification de l'énoncé (ce sont " les circonstances dans lesquelles une expression est employée qui constituent $[$ make] sa signification „ $[B B$, p. 104]). En somme, ce sur quoi s'appuie l'interprète pour traduire les expressions d'une langue étrangère (leur rôle ou usage dans des jeux de langage) est en même temps constitutif de leur signification. Comme le dit Wittgenstein. " [u]n critère [aucuel aurait recours un traducteur] de ce que signifient [par un mot 
les locuteurs d'une langue étrangère] serait les occasions dans lesquelles ils [1" emploient [...], le rôle $[\ldots]$ qu'il joue [...] dans [leur] vie " $(B B$, p. 94) .

La "théorie de la signification-usage " - la conception d'après laquelle la signification d'une expression est non pas un objet, l'objet auquel elle réfère, mais son usage ou son rôle dans des jeux de langage - fournit selon Wittgenstein la solution au probleme de la signification d'expressions telles que "le nombre un "ou les mots "temps" ou "esprit". Il existe beaucoup d'usages des noms de nombres (voir $P U, \S 9$ ), mais un exemple - similaire à celui du jeu de. l'épicerie - en est un dans lequel le constructeur donne des ordres de la forme " $\mathrm{D}$ - briques!" (c’est-à-dire en français " $V$ a me chercher quatre briques!") (voir $P U, \S 8$ ) : pour obéir à l'ordre du constructeur, son aide se rend à l'endroit où sont entreposíes les briques; il énumère à haute voix la série des lettres de l'alphabet jusqu à la quatrième en prenant une brique pour chacune des lettres et il vient les remettre à A. Le traducteur peut inférer de l'observation des « circonstances » du jeu que les mots « a », «b», « $c$ » et « $d$ » sont des noms de nombres dans la mesure où leur rôle dans le jeu (le fail en particulier que $B$ les énumère en prenant un objet pour chaque lettre) diffère entre autres de celui des noms de couleurs (les lettres a $a$ », a b », " $c$ »..., par exemple, ne sont pas inscrites sur un tableau d'échantillons face aux noms de couleurs) ou des noms d'objets (les lettres ne sont pas inscrites sur des étiquettes contrairement au mot " pomme "). L'usage (le rôle d'ensemble dans le jeu) des lettres " a ", “b ». " c "... est " totalement différent "de celui des substantifs. des démonstratifs (voir $P U, \S 8$ ), des adjeclifs référant à des propriétés. cte.

Mais si l'usage d'un mot - ou les données sur l'observation desquelles s'appuic un interprète pour le traduire - est constitutif de sa signification, il semble qu'il s'ensuive une forme de réductionnisme. Wittgenstein compare souvent dans ses écrits les mots d'une langue aux pièces du jeu d'échecs. On pourrait soutenir que, en réponse à la question "En vertu de quoi une pièce est-elle le roi aux échees?" (ou à la question "Qu'est-ce qui est constitutif de l'identité de la pièce. dans le jeu "), on puisse dire que c'est le roi en vertu de l'usage cu'en font les joueurs (usage informé par les règles usuelles du jeu d'échec), par conséquent de son “ rôle " dans le jeu. La réponse à la question "En quoi consiste l'identité d'un certain mot " M" dans la langue (en vertu de quoi " $M$ " a-t-il la signification qu'il possède) ?" serail similaire. Mais si on peut trouver plausible le réductionnisme quant. aux échecs ou aux jeux de langage que Wittgenstein qualifie de

7. Voir la remarque du Cahier brun : « [L]e fait qu'un mot [d'une langue étrangère] se traduise correctement par un $[. .$.$] mot français dépend du rôle que joue le mot$ dans l'ensemble de la vie [des locuteurs], des occasions dans lesquelles il est employé, des expressions d'émotions dont il est généralement accompagné, $[\ldots]$ etc. $\leadsto(B B$, p. 103$)$. 
“ simples », il l'est beaucoup moins pour les langues naturelles. Le " non-réalisme" de Wittgenstein en ce qui concerne le concept de signification prend-il la forme d'un semblable réductionnisme?

Le réductionnisme en question ne serait pas de type béhavioriste. D'un côté, il n'y a aucune raison de penser que les descriptions d'activités sur l'observation desquelles s'appuie le traducteur diffèrent de celles qu'on donnerait dans le langage courant (le traducteur constate, entre autres circonstances qui le justifient à traduire l'énoncé du constructeur par "Apporte-moi une brique! ", que A croit qu'il n'y a plus de briques, qu'il désire que B lui en apporte, ctc.): les descriptions de l'usage renvoient à des états mentaux des locuteurs, à leurs états de croyance, de désir, etc. D'un autre côté, il y a tout lieu de penser que les " circonstances "n'incluent pas seulement les activités des participants au jeu mais également, comme dans l'exemple cité plus haut, le fait qu'un signe ait été peint sur la porte d'une maison (ou que les noms de couleurs figurent sur des tableaux face à des échantillons de couleurs ou le fait (qu'un mot soit inscril sur une étiquette apposée à une armoire) (voir $B B, \mathrm{p} .172 ; P U, \S 37)$.

Par ailleurs, il ne semble pas qu'on doive prendre de façon trop' littérale l'analogie avec les échecs (le rapprochement entre le jeu d'échecs et les formes de langage simples montre sans doute déjà dans l'esprit de Wittgenstein les limites de l'analogie). Une meilleure comparaison serait probablement celle établie entre un mot et un billet de banque (voir $Z, \S 143 ; W L C$, p. 30, 46). On pourrait demander : an vertu de quoi ce bout de papier est-il un billet de cinq dollars? Manifestement, les propriétés physiques de l'objet (ses dimensions, le type d'encre utilisée, etc.) ne sont pas pertinentes (voir $P U, \S 109$ : " nous parlons du langage comme des pièces aux échecs Ior'scu'il s'agit de donner les règles du jeu et non de décrire leurs propriétés physiques n). Ce qui l'est en revanche est le genre d'usage que les gens en font (se procurer des biens, rembourser des emprunts, payer des amendes) au sein d'institutions telles que des commerces, des banques ou des institutions financières. Un " explorateur" (disons un anthropologue venu de Mars) pourrait savoir que c'est un billet de banque et éventuellement savoir que sa valeur est de cinq dollars en observant son "rôle " (sa "fonction ") dans ces transactions, les "circonstances" dans lesquelles les gens l'emploient. Rien d'autre ne semble nécessaire pour savoir qu'il s’agit d'un billet de banque et il est effectivement plausible de soutenir que ces " rôles " sont constitutifs de son identité.

Mais pouvons-nous imaginer une liste exhaustive de toutes les circonstances constitutives de l'identité du billet? Pour qu'il sache qu'il s'agit d'un billet de banque. l'explorateur doit savoir quelles places occupent dans l'économie les banques, les commerces ou les institutions financières ; pour savoir cela, il doit savoir beaucoup de choses sur les comportements et la psychologie des agents économiques (donc en général des êtres humains), sur la vie en 
société, sur nos “formes de vie ", etc. Mais, contrairement à ce que suggère le réductionnisme quant aux billets de banque, il ne semble pas possible de dresser la liste de toutes ces circonstances. On pourrait en dire autant des expressions diune langue naturelle el cest peut-être à cela que fait allusion Wittgenstein quand il écrit (dans un texte que jai déjà en partie cité) : "ce qui caractérise un ordre en tant que tel ou une description en tant que telle $[\ldots]$, etc. est $|. .$.$| le rôle$ que [son] énonciation [...] joue dans la pratique entière du langage " $(B B$, p. 103 ; je souligne). Comme il le dit également plus loin : " [Le| fait qưun mot [d'une langue étrangère] se traduise correctement par un certain mot français dépend du rôle que joue le mot daus lensemble de la vie |de ceux qui parlent la languel" (Ibid. : je souligne). Le réductionnisme en ce qui concerne les expressions de la langue irait à l'encontre de ce " holisme " de lointerprétation el de la signification 8 .

\section{Quelques concepts mentaux dans les Recherches}

On se méprend sur la grammaire ou l'usage des expressions " lo nombre un ", "la signification de “ $I I$ " "ou " la permission de sasseoir sur un siège au cinéma si on suppose quil doil leur correspondre des entités telles que des nombres, des significations ou des permissions pour qu'elles signifient quelque chose. Wittgenstein défend un point de vue similaire au sujet d"un certain nombre de. verbes psychologiques, entre autres les verbes "comprendre " (comme dans l'expression " comprendre un mot "ou "comprendre comment suivre une règle "), "connaître „ (comme dans "connaîtro lalphabet"), "savoir", "croire ", a avoir une intention ", allendre" et "espérer": il n'est pas non plus nécessaire, pour que ces expressions aient une signification, que quelque chose leur corresponde "dans le monde " - ni d'ailleurs dans nos "esprits "

On peut lire à un endroit dans les Recherches: "Quand on dit que: connaître l'alphabet est un état de l'esprit, on pense à l'ćlat d'ur appareil mental (peut-être du cerveau) au moyen duquel nous expliquons les manifestations de ce savoir. Nous appelons un tel état une disposition " $(P U, \S 149)$. On lit ensuite : on peul s'objecter à ce que. l'on puisse parler ici d'un état de l'esprit dans la mesure où il devrait y avoir deux critères pour un tel état, en l'occurrence la connaissance de la construction de l'appareil, mise à part [la question de savoir quels en sont] les effets (Jbid.).

S'il y a des objections à dire que connaître l'alphabet est un « état de l'esprit ", en quel sens ?' Apparemment au sens où on serait tenté de dire que connaitre l'alphabet est un état conscient. Wittgenstein écrit : "Comprendre un mot" : un état. Mais un état mental? Nous appelons la dépression, l'excitation, la douleur des états mentaux *

8. Sur le " holisme " de Wittgenstein, voir mon article "Interprétation, signification et " usage" chez Wittgenstein " (Dialogue, vol. 35, 1996). 
$(P U$, p. 59). Les états de conscience ont en effet une durée (on peut ressentir une douleur pendant quinze secondes ou être déprimé pendant deux jours), contrairement à la compréhension d'un mot ou à la connaissance de l'alphabet (il serait étrange de dire " Il a su ce que le mot “ $\mathrm{M}$ " signifie pendant une demi-heure $m$ ). Comprendre un mot serait un état, mais un état d'un type différent de celui de la dépression, de l'excitation ou de la douleur. À un autre endroit dans les Recherches, on lit : " L'attente est grammaticalement un état ; tel que : être d'une certaine opinion, espérer quelque chose, savoir quelque chose, être capable de faire quelque chose " $(P U, \S 572)$. Au paragraphe suivant, on lit : Avoir une opinion est un état. - De quoi est-ce un état? De l’âme? De l'esprit? Eh bien, de quoi est-ce que l'on dit que cela a une opinion? De monsieur N. N. par exemple. EL c'est la réponse correcte " $(P U, \S 573)$. La grammaire (au sens de la grammaire usuelle) compte l'attente, l'opinion, l'intention, le savoir, ete. comme des états (on ne désigne pas l'attente ou l'opinion au moyen de verbes d'actions ou d'activités), done des états disposilionnels et non des états de conscience comme la dépression ou la douleur (voir $P U, \S 149$ ).

Mais il ne semble pas que ce soit cela que veut dire Wittgenstein. Il entend nier dans le paragraphe 149 non pas que connaître l'alphabet est un état mental conscient (bien qu'il refuse aussi cette idée comme le montrent les autres passages que je viens de citer); ce qu'il veut plutôt nier est que l'expression "connaitre l'alphabet" semploie pour désigner un état quel qu'il soit : grammaticalement (au sens de la grammaire courante), connaître l'alphabet est sans aucun doute un état et il est parfaitement correct de dire que c'est l'état d'une personne (voir $P U, \S 573$ cité ci-dessus), mais dire cela ne signifie pas qu'il s'agit d'un état en vertu de l'existence d'un autre état yui serait celui de lappareil mental (de l'esprit. de l'âme) ou du cerveau de la personne à laquelle on l'attribue. C'est ce qu'il soutient également au sujet d'un certain nombre de verbes psychologiques d'activités ou de processus, entre autres "comprendre "au sens d'une compréhension soudlaine (voir $P U, \$ 151-155$ ), "lire " (voir $P U$, $\S 156-171$ ) el "se souvenir" (voir $P U, \S 305-306$ ). Lierreur est semblable à celle commise au sujet des mols "nombre », " temps " ou "esprit": on traite les verbes psychologiques d'états ou de processus sur le modèle des verbes d'états ou de processus ordinaires et on conçoit leur usage (leur" "grammaire ») à partir de l'analogie avec ces autres verbes : on suppose de façon erronée que si, dans le cas des verbes d'états on de processus ordinaires, il existe des états ou processus auxquels les verbes réfèrent, cela doit être vrai également des verbes psychologiques " comprendre ", "savoir ", "croire " ou "avoir une intention". Je citerai des textes à l'appui de cette interprétation.

Il existe des verbes dispositionnels (ou de capacité) qui servent à décrire des états ou processus qu'il est possible d'observer'; il leur 
correspond, dit Wittgenstein, une "expérience sensorielle stationnaire » $(B B$, p. 101) : on dit d"un objet ou d'un mécanisme qüil peut se comporter de telle ou telle façon (avoir tels ou tels effets) parce quion peut observer l'état de l'objet ou du mécanisme en question (il donne dans le Cahier brun l'exemple d'un dispositif constitué d'une pièce de bois dotée d'une rainure circulaire dans laquelle on a inséré une tige : l'énoncé a La tige peut glisser le long de la rainure en décrivant un cercle » rapporte l'état du dispositif [voir $B B, p .100]$ ). Dautres verbes dispositionnels ne rapportent pas l'existence d'états qu'on peut observer ; il ne leur correspond aucune "expérience sensorielle qui dure aussi longtemps que dure l'état ". " $\mid$ A $\mid$ u lieu de cela ", dit Wittgenstein, "le critère définissant |defining criterion| pour' que quelque chose soit dans cet état consiste en certains tests " (BB, p. 101) : on vérifie que l'objet satisfait des énoncés de la forme "Si telle ou telle condition est réalisée. l"objet se comporte de telle ou telle façon". Cependant, même dans des cas comme ccux-là, le langage, nos formes d'expressions elles-mêmes suggèrent l'existence. d'un état de l'objet (d'une structure, d'un mécanisme) qu'est supposé désigner le verbe : " nous sommes fortement enclins à employer la métaphore de quelque chose qui se trouve dans un certain état pour [exprimer le fait] que quelque chose peut se comporter d"une façon particulière. Et cette manière de se représenter les choses, ou cette métaphore, est renfermée dans [embodied in l'expression "II esI capable de..." " $[\ldots] »(B B$, p. 117). Dans un énoncé dispositionnel, "le verbe est employé au présent, suggérant que les expressions sont des descriptions d'états qui existent au moment oì l"on parle " (Ibid.). Comme il le dit également dans l'un de ses cours : "Les énoncés dispositionnels sonı toujours fondamentalement des énoncés au sujel d'un mécanisme et ont la grammaire lau sens de la grammaire usuelle] d'énoncés au sujet d'un mécanisme. |... Dans un nombre considérable de cas, nos mots ont la forme d'énoncés dispositionncls référant à des mécanismes peu importe qu’un mécanisme existe ou non " $(W L C$, p. 91). Et c'est cela qui incite à voir dans les verbes psychologiques dispositionnels des expressions employées pour référer à des états ou processus ayant leur siège dans l'esprit ou le cerveau :

La même tendance se montre dans le fait que nous appelons la capacité de résoudre un problème mathématique, la capacité d'apprécier une pièce de musique, [d'énumérer les lettres de l'alphabet, etc.] des états cle l'esprit; nous ne: voulons pas dire par cette expression " phénomènes mentaux conscients ". Un état de l'esprit en ce sens est plutôt l'état d'un mécanisme hypothétique, un nodè̀le de. l'esprit censé expliquer les phénomènes mentaux conscients. [...] De la même manière, nous ne pouvons nous empêcher de concevoir la mémoirc comme une sorte d'entrepôt. Notez également la certitude qu'ont les gens qu'à la capacité d'additionner, de multiplier ou de réciter un poème par cour, etc., il doit correspondre un état particulier du cerveau de la personne, bien qu'ils ne sachent par ailleurs rien, à toutes fins utiles, au sujet de telles correspondances psychophysiologiques. Nous regardons ces phénomènes /additionner, multiplier, 
énumérer les lettres de l'alphabet] comme les manifestations de ce mécanisme et leur possibilité est la construction particulière du mécanisme lui-même. ( $B B$, p. 117-118)

La grammaire (au sens cette fois de la grammaire philosophique) des verbes psychologiques de capacités est en fait différente "totalement différente", dirait Wittgenstein - de celle des verbes dispositionnels référant à des états ou à des mécanismes. Pour revenir au paragraphe 149 des Recherches cité plus haut, si connaître l'alphabet élail un état qui explique causalement ses "effets" (les comportements des indiviclus), si, comme dit Wittgenstein (voir PU, $\$ 146$ ), il en était la " source », on pourrait avoir recours à deux types de critères pour l'attribuer à quelqu'un, à savoir " la connaissance de la construction de l'appareil " (de la structure particulière du mécanisme) el le comportement (réciter l'alphabet). Mais ce n’est pas le cas : il est clair à examiner sa grammaire que l'expression n'est pas altribuée sur la base d'un critère du premier type. Le critère (au sens d'un "critère définissant ") de la connaissance de l'alphabet est plutôt relatif au comportement (au fait qu'un individu récite l'alphabet). Au sujet du concept de compréhension (entenduc ici au sens d'un processus et non (l'un état). Wittgenstein écrit : "Essayez de ne pas penser du tout à la compréhension comme à un "processus mental ". - Car c'est cette expression qui crée chez vous la confusion. Demandez-vous plutôt : dans quel cas, dans quel genre de circonstances $[$ Umstä̈nden| disons-nous [que quelqu un comprend] „ $(P l$, $\$ 154)$. Par les " circonstances ", il faut comprendre non seulement les comportements pris au sens étroit consistant à réciter l'alphabet ou à poursuive une suite de nombres, mais le fait par exemple qu'unc personne ait appris l'alphabet à l'école. le fait qu'on lui ait enseigné l'algèbre, qu'elle ait utilisé des formules semblables à d'autres occasions, etc. (voir $P U, \S 179 ; B B$, p. 114).

In autre passage des Recherches reprend la même idée en citant l'exemple des verbes "attendre ", "croire " et "espérer". On lit ceci : "Demandez-vous: qu'est-ce que cela veut dire" croire que te théorème de Goldbach est vrai "? En quoi consiste cette croyance ?" (PU, \$ 578). Croire que le théorème est vrai, répond Wittgenstein. e'est. entre autres choses, chercher à en découvrir une preuve; si on sail comment s'y prendre pour la découvrir. "nous [savons] à quoi cela revient que de croire à cette proposition "(Ibid.). Ainsi. la recherche dine preuve compte au nombre des " circonstances" de la croyance et elle est constitutive de cette croyance. Au sujet du verbe "attendre (comme dans l'expression " attendre que se produise une explosion "), il écrit: "Liattente est insérée dans une situation [Situation], de laquelle elle surgit. L'attente d'une explosion peut surgir par exemple d'une situation dans laquelle on s'attend à ce que se produise une explosion” $(P U, \S 581)$. Et, à propos du verbe " espérer" ", il fait remarquer qu'on peut dire d"une personne qu'elle espère la venue de quelqu'un qui va lui apporter de largent clans 
certains types de situations seulement, dans un cadre (Umgebung) ou un contexte (Zusammenhang) particulier où l"institution de l'argent existe, où on sait que la personne a un besoin pressant d'argent, où elle croit que telle ou telle personne peut hi en apporter, clc. (voir $P U, \S 584)$. Quand les circonstances changent, il n'y a plus lieu parfois de parler d" attente ", de "croyance "ou d' "cspoir". (Dans un contexte différent du contexte familier, dit Wittgenstein, la cérémonie de couronnement d'un roi pourrait avoir une signification complètement différente de celle qu’clle a habitucllement - ou n'avoir aucune espèce de signification - si elle a lieu chez un peuple pour lequel l'or est un métal peu dispendieux, oì le lissu de la robe du roi est facile à confectionner, etc. (voir $P U, \S 584$ )

On pourrait encore exprimer la même idée comme suit : de même que pour traduire un énoncé d'une langue étrangèrc un interprètc doit prendre en compte les circonstances dans lesquelles il est fait -. son rôle d'ensemble dans le jeu (voir section 2) - il (loit tenir compte de la "situation" ou du "contexte" dans lequel unc personne développe (par exemple) une série de nombres pour (jü soit justifié à dire qu’elle comprend une certaine formule algébrique (pour qu'il soit justifié à dire qu elle espère qu'on lui apporte de l'argent, qu'elle s'attend à une explosion, etc.). Le critère pour ćtablir qu'un mot d'une langue étrangère se traduit correctement par un certain mot français est la similitude des rôles dans lés deux langues; le critère pour dire si quelqu'un comprend unc formule algébrique est le fait que la personne ait appris l'algèbre à l'ćcole, 'qu'elle ait montré qu'elle sait appliquer d'autres formules et ainsi de suite - et non le fait qu'on ait observé quelque chose dans son esprit ou son système nerveux qui serait la source de cette compréhension (notre critère pour dire qu'un piano mécanique " lit "correctement ou non une partition est, dit Wittgenstein, l'étal de son mécanisme ; cependant on n'emploie pas un critère de ce genre pour dire si un être humain est réellement en train de lire et non de prononcer des mots au hasard [voir $P U, \$ 157])$. D'autre part, de même que ce sur quoi s'appuie l'interprète pour traduire les énoncés de la langue - leur rôle ou leurs circonstances d'emploi est constitutif de leur signification (voir section 2), les circonstances sur l'observation desquelles on s'appuie pour dire qu'une personne comprend ou qu'elle a une certaine intention sont constitutives de sa compréhension ou de son intention. C'est ce qu'on peut lire dans une des Remarques sur la philosophie de la psychologie:

Si quelqu'un dit : «Je crois que, lorsque j'entends un mot que je comprends, je ressens toujours quelque chose que je ne ressens pas quand je ne comprends pas le mot - - il fait un énoncé sur son expérience particulière. Quelcu'un d'autre a peut-être une expérience complètement différente de la sienne ; tet si les deux font un usage correct du mot " comprenclre ", l'cssence de la compréhension réside dans cet usage et non dans ce qu'ils disent sur leurs expériences. (BPPI, § 212) 
Ce que quelqu'un dit ressentir lorsqu'il comprend - ce qui se passe, qu'il en ait conscience ou non, dans son esprit ou son cerveau - n'est pas ce qui importe quand on se demande en quoi consiste sa compréhension ; l'important (ce qui est constitutif de la compréhension) est l'usage que les deux locuteurs font du verbe " comprendre ": les circonstances (la situation, le contexte) dans lesquelles eux-mêmes ou d'autres locuteurs disent et sont justifiés à dire qu'ils ont compris.

Ici à nouveau se pose le problème du réductionnisme et du béhaviorisme - le problème dont il était question plus haut à propos du concept de signification. Il semble d'un côté que, bien que Wittgenstein dise que la compréhension, l'attente ou la croyance sont des états d'une personne (au sens de la grammaire courante), il nie quion puisse les assimiler à des élats internes de l'esprit ou du cerveau, ce qui, semble-t-il, implique une sorte de béhaviorisme "éliminationniste „ (qui revient à soutenir qu'il n'y a rien de tel que des états mentaux et que seuls existent les comportements des individus). De l'autre, s'il n'est pas béhavioriste en ce sens-là, il pourrait être un "béhavioriste logique" du type réductionniste et non éliminationniste qui considère que les comportements sont constitutifs des états mentaux (en ce sens qu’ils s'y réduisent).

Il ne semble pas y avoir de raison de croire que Wittgenstein est un béhavioriste du type éliminationniste. Il y a une supposition, dit Wittgenstein, qui conduit à nicr l'existence des états et processus mentaux qui est à la source du béhaviorisme éliminationniste. unc supposition qui " passe totalement inaperçue " et qui semble la plus " innocente " (PU, §308), la supposition que la compréhension. l'attente, la croyance, etc. sont des états ou des processus semblables aux ćtats ou processus ordinaires, bien qu'on ne soit pas en mesure de dire quoi que ce soit à leur sujet : "Nous [c'est-à-dire les philosophes tentés par cette variété de béhaviorisme| parlons de processus et d'états et laissons indéterminée leur nature. Un jour, peut-être, nous en saurons plus à leur propos - pensons-nous "(Ibid.). Mais une telle supposition entraine avec elle " une façon particulière de voir les choses [car| nous avons un concept bien défini de ce que cela veut (lire : connaître de plus près un processus »(Ibid.). On n a cependant aucune idée de la façon dont il faudrait s'y prendre pour en savoir plus sur la compréhension, l'attente ou la croyance (considérées comme des élats internes). puisque personne ne peut dire ce qui se passe dans le cerveau de quelqu'un qui comprend, attend ou croit quelque chose. De la sorte, "la comparaison avec les états ou processus observables] qui devait nous permettre de nous expliquer à nous-mêmes nos pensées s'effondre » (Ibid.). Et il semble que nous devions en conclure que la compréhension. l'attente, etc. n'existent pas ( Nous devons [...] nier [l'existence du] processus non encore compris dans le médium [le médium de l'esprit] non encore exploré » [Ibid.]). En somme, soit la compréhension, la remémoration, etc., sont 
des processus internes, soit elles n'existent tout simplement pas : pour qu'elles existent, elles doivent pouvoir faire l'objet du même type d'investigation (physiologique, chimique, etc.) que toul autre processus. Comme ce n'est pas le cas, le béhavioriste en conclul que la compréhension ou la remémoration n'existent pas : on ne devrait en définitive parler que des comportements.

En réponse à cela. Wittgenstein nie " que limage du processus mental nous donne une idée exacte de l'usage [des mots| "se. souvenir" [" comprendre ", ete. $\mid$ ". Car " [...] cette image avec ses ramifications nous empêche de voir l'usage [de ces mots| comme il est” $(P U, \S 305)$. On lit également dans la Grammaire philosophique: a Il en est du processus psychique de la compréhension comme de l'objet arithmétique trois. Le mot " processus n ici et le mot « objet » là induisent une attitude grammaticale erronée vis. à-vis du mot " $(P G$, p. 85). Les "usages" (la " grammaire philosophique ») des verbes psychologiques dispositionnels diffèrent de ceux des verbes non psychologiques référant à des états ou à des processus : le concept de compréhension n'est pas le concept d'un quelque chose supposé avoir son siège dans l"esprit ou le cerveau dune personne qui serait la source (au sens où il fournirait l'explication causale) de ses comportements. Il n'y at donc pas lieu de nier que les gens comprennent des choses (se souviennent, elc.) en sappuyant comme le béhavioriste éliminationniste sur le fait que l'on ne sait pas ce qui se passe dans le cerveau d'une personne quand, sur la base des critères courants, on dit qu'elle comprend.

Wittgenstein est-il un béhavioriste au second sens, c'est-à-dire un béhavioriste logique? On peut remarquer d'abord, comme plus haut à propos du concept de signification, que ce ne sont pas selon lui que les comportements, mais également les "circonstances" qui sont constitutifs des états ou processus mentaux. Il écril par exemple : " $[\ldots]$ qu'est-ce qu'inclut ici le comportement fquand on parle de la pensée, de la colère, de l'espoir, de la peur, de l'intention]? Seulement le jeu de l'expression du visage et les gestes? Ou bien également le cadre |Umgebung|, pour ainsi dire: l'occasion $[$ Anlass] qui donne lieu à cette expression ?" " $(R P P I, \S 129$; voir aussi $R P P I I, \S 166)$. D'autre part, il suggère à au moins un endroit dans les Recherches qu'il n'est pas possible d'isoler une " totalité de conditions » telles que, si elles étaient réalisées, une personne ne pourrait pas ne pas comprendre ou se remémorer quelque chose $(P U$, $\S 183$; voir $B B$, p. 114). Il rejette donc probablement aussi la composante réductionniste du béhaviorisme logique.

En résumé, il y a pour l'auteur des Recherches des expressions dans la langue qui désignent des choses (celles qu'on peut définir entre autres au moyen de définitions ostensives), des substantifs qui réferent à des objets, des verbes auxquels correspondent des états, activités ou processus et des énoncés qui décrivent des états de choses. D'autres expressions ne remplissent pas une fonction 
référentielle; c'est le cas des expressions "le nombre un ", "la signification de " $M$ " " ou " la permission de s'asseoir sur un siège au cinéma ". C'est vrai également d'un certain nombre de verbes psychologiques tels que " comprendre ", " connaître ", " savoir ", " croire ", " avoir une certaine intention ", "attendre " ou " espérer ". En dépit de ce que suggère la grammaire courante, ces verbes n'ont pas pour fonction de référer à quelque chose, ni à des états ou processus ayant leur siège dans le système nerveux, ni à des états ou processus hypothétiques de l'“ appareil psychique ». On peut ainsi décrire sa position comme une forme de "réalisme "vis-à-vis certains types d'objets (d'états ou de processus) auxquels réfèrent les mots de la langue courante (les tables, les crayons, les états et processus observables, etc.) el une forme de "non-réalisme " quant aux nombres, au temps, aux esprits et, en particulier, aux significations et à un certain nombre d'états et de processus mentaux. Bref, il semble que Wittgenstein ait conscrvé durant la seconde phase de sa vic philosophique des préoccupations semblables à celles qu'il avait eues plus tôt concernant les implications ontologiques du langage ${ }^{9}$.

Département de philosophie

C'égep de Saint-Hyacinthe

9. Les recherches en vue de la rédaction de cet article ont été rendues possibles grâce à une subvention du FCAR auquel je tiens ici à exprimer ma reconnaissance. 